\title{
Abordaje anestésico en cirugía torácica asistida por robot: estudio de cohorte unicéntrico
}

\author{
Anaesthetic approach in robot-assisted thoracic surgery: a single-center, \\ cohort study
}

David Álvarez-Villegas MD. ${ }^{1, *}$, Elena Campistol MD. ${ }^{1}$, Guillermo Puig MD. ${ }^{1}$, Anna Ureña MD, PhD. ${ }^{2}$, Miguel Ángel Delgado MD. ${ }^{1}$, Sebastián Videla MD, PhD. ${ }^{3}$, Marc Giménez-Milà MD, PhD, EDAIC, EDIC 1.

Servicio de Anestesiología y Reanimación. Hospital Universitari de Bellvitge, L’Hospitalet de Llobregat. Barcelona, España.

Servicio de Cirugía Torácica. Hospital Universitari de Bellvitge. L'Hospitalet de Llobregat. Barcelona, España.

3 Unidad de Soporte a la Investigación Clínica. Servicio de Farmacología Clínica. Hospital Universitari de Bellvitge. L'Hospitalet de Llobregat. Barcelona, España.

Financiación y conflictos de Interés: La presente investigación no ha recibido ayudas específicas provenientes de agencias del sector público, sector comercial o entidades sin ánimo de lucro.

Los autores declaran no tener conflictos de interés.

Fecha de recepción: 13 de abril de 2021 / Fecha de aceptación: 25 de mayo de 2021

\begin{abstract}
Introduction:_Anaesthesia for robot-assisted thoracic surgery (RATS) has some peculiarities that can impact on the development of perioperative complications. The objective of our work is to report our experience on the perioperative approach of RATS, focusing on clinical outcome variables. Material and Methods: A single-center, retrospective, cohort study including patients operated via RATS between January 2019 and February 2020. Demographic data, type of surgical intervention, invasive intraoperative monitoring, anaesthetic and analgesic approach, hospital and critical care unit (UCC) length of stay, need for readmission to UCC, postoperative analgesia, pain assessment with the NPRS scale after 1, 24 and 48 hours after surgery and postoperative complications during admission were collected. Results: A total of 73 patients were analysed. Serratus anterior plane block was associated with a lower consumption of fentanyl compared with the rest of analgesic techniques ( $p$-value $=0.01$ ). All patients were extubated in the operating room. 48 patients $(65.7 \%)$ were transferred to the UCC. $3(4.1 \%)$ were discharged at home the same day of procedure. The mean postoperative pain at 1, 24 and 48 hours postoperatively was 2.8 (SD 2.6), 1.7 (SD 1.8) and 1 (SD 1.4). Nausea and persistent postoperative leak were the most frequent complications. Conclusions: RATS seems to be a safe technique that allows a short hospital length of stay and, in some cases, an outpatient circuit surgery. Serratus anterior plane block reduced the intraoperative fentanyl requirements in our population.
\end{abstract}

Key words: Robot-assisted thoracic surgery, intraoperative analgesia, multimodal analgesia, perioperative complications.

\section{RESUMEN}

Introducción: La anestesia en cirugía torácica asistida por robot (RATS) tiene unas particularidades que pueden impactar en la aparición de complicaciones perioperatorias. El objetivo de nuestro trabajo es aportar nuestra experiencia sobre el abordaje perioperatorio de la RATS, focalizados en variables de resultado clínico. Material y Métodos: Estudio de cohorte, retrospectivo y unicéntrico donde se incluyeron los pacientes intervenidos mediante RATS entre enero de 2019 y febrero de 2020 . Se recogieron datos demográficos, tipo de intervención quirúrgica, monitorización invasiva intraoperatoria, técnica anestésica y analgésica, estancia hospitalaria y en cuidados críticos (UCC), necesidad de reingreso en UCC, analgesia postoperatoria, valoración del dolor por la escala NPRS pasadas 1, 24 y 48 h de la intervención y complicaciones posoperatorias. Resultados: Se analizaron un total de 73 pacientes. Aquellos en quienes se realizó el bloqueo del plano serrato anterior requirieron un menor consumo de fentanilo comparado con el resto de las técnicas analgésicas ( $p$-valor $=0,01$ ). Todos los pacientes fueron extubados en el quirófano. 
Cuarenta y ocho $(65,7 \%)$ fueron trasladados a la UCC. Tres $(4,1 \%)$ fueron dados de alta el mismo día en régimen de CMA. La media de dolor posoperatorio, a la 1, 24 y 48 h postoperatorias fue de 2,8 (DE 2,6), 1,7 (DE 1,8) y 1 (DE 1,4). Las náuseas y la fuga persistente posoperatoria fueron las complicaciones más frecuentes. Conclusiones: Entendemos la RATS como una técnica segura, que permite una corta estancia hospitalaria y, en algunos casos, un circuito ambulatorio. El bloqueo del plano serrato anterior disminuyó los requerimientos de fentanilo intraoperatorio en nuestra población.

Palabras clave: Cirugía torácica robótica, analgesia intraoperatoria, analgesia multimodal; complicaciones perioperatorias.

\section{Introducción}

a evolución de la cirugía torácica se dirige hacia una progresiva disminución de la agresión quirúrgica con el auge de la cirugía videotoracoscópica (VATS) y recientemente la aparición de cirugía asistida por robot (RATS)[1]-[3]. La menor agresión tisular con incisiones de menor tamaño, así como una mayor precisión quirúrgica debido a la asistencia robótica, son atributos que se han sugerido a esta técnica. A pesar de ello, muy poco es lo escrito en cuanto al manejo anestésico y analgésico en estas modalidades quirúrgicas mínimamente invasivas, especialmente en la RATS.

El adecuado control del dolor cobra especial importancia en los pacientes de cirugía torácica por distintos motivos, como son: permitir la extubación en quirófano, evitar la aparición de atelectasias, facilitar la correcta expansión pulmonar y reducir la aparición de dolor crónico postoperatorio[4],[5] favoreciendo la implementación de un protocolo de recuperación acelerada tras la cirugía[6] además de aportar el máximo confort posible al paciente. Del mismo modo, cabe reseñar los potenciales riesgos de este abordaje; la inyección de anhídrido carbónico $\left(\mathrm{CO}_{2}\right)$ que se realiza durante el procedimiento para conseguir un espacio suficiente para poder operar al paciente, puede inducir hipercapnia, así como compromiso hemodinámico[7]. La compresión mediastínica y la hipercapnia con el aumento de resistencias pulmonares son plausibles causas de hipotensión intraoperatoria. La severidad de la hipercapnia, así como las características del paciente (presencia de hipertensión pulmonar e hipertensión endocraneana) influirán en la capacidad de tolerancia y la necesidad de disminuir la presión de insuflación del $\mathrm{CO}_{2}$.

El objetivo de nuestro trabajo es, por lo tanto, aportar datos acerca del manejo anestésico, tanto intraoperatorio como posoperatorio, así como variables de resultado clínico obtenidos durante el primer año de implementación del programa de cirugía torácica robótica para resección de tumores pulmonares y lesiones mediastínicas en un hospital universitario del territorio español.

\section{Material y Métodos}

\section{Diseño del estudio}

Se trata de un estudio de cohorte, retrospectivo y unicéntrico. Éste fue aprobado por parte del Comité de Ética de Investigación Clínica del Hospital Universitario de Bellvitge (código ICPS008/20) eximiendo la necesidad de obtención del consentimiento informado de los participantes. Este estudio se llevó a cabo siguiendo los principios éticos básicos contenidos en la
Declaración de Helsinki (Revisión de la 64ª Asamblea General, Fortaleza, Brasil, octubre de 2013), la Buena Práctica Clínica y las normativas aplicables. Los datos de los pacientes fueron tratados con confidencialidad asegurando su anonimato de acuerdo con el Reglamento (UE) 2016/679 del Parlamento Europeo y de Consejo de 27 de abril de 2016 relativo a la protección de las personas físicas con respecto al tratamiento de datos personales y a la libre circulación de estos datos, así como al resto de leyes y normativa vigente y aplicable, como la Ley Orgánica $3 / 2018$, de 5 de diciembre, de protección de datos personales y garantía de los derechos digitales.

\section{Población del estudio}

Se incluyeron todos los pacientes intervenidos mediante RATS entre enero de 2019 y febrero de 2020. Los criterios de inclusión fueron pacientes mayores de 18 años de ambos sexos intervenidos en este período de tiempo por RATS, mientras que los criterios de exclusión fueron la pérdida o ausencia de datos a analizar y la reconversión de la técnica quirúrgica por cualquier causa. La obtención de los datos se realizó mediante la información registrada en plataforma electrónica y en la gráfica de anestesia.

\section{Técnica anestésica}

Como paso previo a la inducción anestésica los pacientes se monitorizaron con pulsioximetría, registro de electrocardiograma con las derivaciones II y V5, presión arterial no invasiva, así como índice biespectral $\left(\mathrm{BIS}^{\circledR}\right)$. Se procedió a la inducción anestésica mediante propofol, fentanilo y rocuronio. Tanto la anestesia inhalatoria con sevofluorano como la endovenosa pura (TIVA) se usaron para el mantenimiento anestésico. Se adecuó la monitorización invasiva según las comorbilidades del paciente y el tipo de procedimiento quirúrgico. Para la ventilación unipulmonar, se empleó ventilación protectora con un volumen corriente de $4-5 \mathrm{~mL} \cdot \mathrm{kg}^{-1}$ y aplicación de presión positiva teleespiratoria (PEEP) de $5-8 \mathrm{~cm} \mathrm{H}_{2} 0$, y $6-7 \mathrm{~mL} \cdot \mathrm{kg}^{-1}$ más PEEP en los momentos de ventilación bipulmonar.

Se trató de mantener la normotermia del paciente empleando unidad de calentamiento por aire forzado durante el acto quirúrgico.

\section{Técnica analgésica}

La analgesia locorregional se realizó tras la inducción anestésica, con el paciente posicionado en decúbito lateral, empleándose como parte de la analgesia multimodal previa a la incisión quirúrgica. Las técnicas realizadas fueron la infiltración intercostal (I) por parte del equipo quirúrgico, bloqueo ecoguiado del plano serrato anterior $(S)$ y del plano erector espinal $(E)$ y en algunas ocasiones combinándose la infiltración de los in- 
tercostales con los bloqueos ecoguiados $(S+l$ o $E+l)$. El bloqueo $S$ se practicó a nivel del $5^{\circ}$ espacio intercostal en la línea axilar media y el bloqueo $E$ se realizó a nivel de T5. Se empleó una aguja de 22 Gauge, (Temena ${ }^{\circledR}$ USC 50 Evolution), administrando un bolo único de $20 \mathrm{~mL}$ de una mezcla de anestésico local basada en mepivacaína $1 \%$ y ropivacaína 0.4\%. Se empleó el fentanilo de forma intraoperatoria para tratar el dolor según el criterio del anestesiólogo.

\section{Destino posoperatorio}

En función de las comorbilidades del paciente, del tipo de procedimiento quirúrgico y de las eventuales complicaciones intraoperatorias acontecidas, tres circuitos eran los posibles en cuanto al destino postoperatorio del paciente: 1 . ingreso en la unidad de cuidados críticos postquirúrgicos (UCC); 2. unidad de recuperación posanestésica (URPA) para ingresar posteriormente en planta de hospitalización; 3. URPA para entrar en circuito de cirugía mayor ambulatoria con alta a domicilio el mismo día del procedimiento quirúrgico (CMA).

\section{Analgesia posoperatoria}

En la URPA y UCC, los pacientes recibieron analgesia pautada basada en AINEs y opioides de rescate, en forma de bolos endovenosos de morfina o PCA de morfina para obtener NPRS menor de 4.

Para el manejo del dolor de los pacientes que entraron en circuito de CMA, tras ser dados de alta de la URPA, se emplearon AINEs, paracetamol y tramadol como fármaco de rescate.

\section{Variables de estudio}

Se recogieron los datos demográficos, el tipo de intervención quirúrgica, la monitorización invasiva intraoperatoria, la técnica anestésica y analgésica, la estancia en UCC y hospitalaria, la necesidad o no de reingreso en UCC, el uso de mórficos en planta.

La valoración del dolor agudo postoperatorio se llevó a cabo por enfermería de UCC, URPA y planta de hospitalización convencional. Se midió el dolor mediante la escala numérica de puntuación del dolor [NPRS por sus siglas en inglés (0: ausencia de dolor; 10: peor dolor imaginable)] a la respiración profunda tras 1, 24 y 48 h de la cirugía.

Se recogieron las complicaciones postoperatorias durante el ingreso del paciente en el hospital.

\section{Análisis estadístico}

Los datos categóricos se presentan como n (\%) y los datos continuos como media [desviación estándar (DE)]. Se utilizó el test de Kruskal-Wallis para comparar datos no paramétricos entre los grupos. Las comparaciones de proporciones se realizaron mediante pruebas de chi-cuadrado o exacta de Fisher. La prueba t de Student o la prueba U de Mann-Whitney y la prueba de chi-cuadrado de Pearson se utilizaron para realizar comparaciones univariadas entre grupos de variables cuantitativas y cualitativas, respectivamente. Para todos los análisis, se consideró significativo un valor de $p$-valor bilateral $<0,05$. Todos los análisis estadísticos se realizaron utilizando STATA/IC 15.1A.

Se siguieron las guías para informes de estudios observacionales de la Strengthening the Reporting of Observational Studies in Epidemiology (STROBE) (8).

\section{Resultados}

\section{Características basales}

Del total de 78 pacientes intervenidos por RATS en el período observado, 5 fueron excluidos; en 4 de ellos se tuvo que reconvertir la técnica a toracotomía, en 2 ocasiones por adherencias y en otras 2 ocasiones por hemorragia, mientras que uno de ellos fue excluido por pérdida o ausencia de datos a analizar. Así, los resultados presentados corresponden a un total de 73 pacientes. La media de edad fue de 62,8 años (DE 12,7). La muestra comprendió 40 hombres (54,8\%) y 33 mujeres (45,2\%) (Tabla 1).

Por tipo de procedimiento, las intervenciones quirúrgicas realizadas fueron: lobectomía en 33 (45,2\%), timectomía en $19(26 \%)$, resección en cuña en $17(23,3 \%)$, timectomía con resección en cuña en $2(2,7 \%)$, lobectomía y resección en cuña en $1(1,4 \%)$ y resección de lesión pleural en 1 paciente $(1,4 \%)$.

\section{Técnica anestésica}

En 66 pacientes $(90,4 \%)$ se realizó mantenimiento con sevofluorano y en 7 pacientes (9,6\%) con TIVA. En 73 pacientes (100\%) se empleó fentanilo y en $3(4,1 \%)$, remifentanilo junto con fentanilo como analgésicos opioides intraoperatorios.

Como técnica de ventilación unipulmonar, se realizó en 72 pacientes $(98,6 \%)$ intubación con tubo de doble luz con comprobación clínica mediante auscultación y guiada por fibrobroncoscopio y en $1(1,4 \%)$ con bloqueador bronquial (tipo Cohen) por vía aérea difícil no prevista.

En cuanto a la monitorización invasiva, se cateterizó un acceso venoso central únicamente en 1 paciente $(1,4 \%)$, mientras que se procedió al sondaje vesical de 44 pacientes $(60,2 \%)$. En 48 casos $(65,8 \%)$ se monitorizó la presión arterial invasiva mediante catéter radial. En sólo 3 pacientes operados de timectomía (15,8\%) se monitorizó la presión arterial invasiva (Tabla 2). No se empleó en ningún caso infusión de agentes vasoactivos durante el acto quirúrgico.

\section{Técnica analgésica}

Las técnicas de analgesia regional empleadas fueron / en 31

\begin{tabular}{lc}
\hline & Tabla 1. Descripción de la muestra $(\mathbf{n}=\mathbf{7 3})$ \\
\hline Edad & años \\
Media (DE) & $62,8(12,7)$ \\
Género & $\mathbf{n}(\%)$ \\
Hombres & $40(54,8 \%)$ \\
Mujeres & $33(45,2 \%)$ \\
ASA & $\mathbf{n}(\%)$ \\
I & $2(2,7 \%)$ \\
II & $45(61,6 \%)$ \\
III & $25(34,3 \%)$ \\
IV & $1(1,4 \%)$ \\
IMC & $\mathbf{K g} / \mathbf{~ m}^{\mathbf{2}}$ \\
Media (DE) & $27,3(4,4)$ \\
\hline
\end{tabular}

DE: desviación estándar; ASA: clasificación de la American Society of Anesthesiology; IMC: índice de masa corporal. 


\begin{tabular}{lcccc}
\hline \multicolumn{5}{c}{ Tabla 2. Monitorización invasiva por tipo de intervención quirúrgica } \\
\hline & $\mathbf{n}$ & PAI (\%) & SV (\%) & CVC (\%) \\
Timectomía & 19 & $3(15,8 \%)$ & $4(21 \%)$ & 0 \\
Lobectomía & 33 & $33(100 \%)$ & $29(87,9 \%)$ & 0 \\
Resección en cuña & 17 & $9(52,9 \%)$ & $8(47,1 \%)$ & $1(5,9 \%)$ \\
Lobectomía + resección en cuña & 1 & 0 & $1(100 \%)$ & 0 \\
Timectomía + resección en cuña & 2 & $2(100 \%)$ & $2(100 \%)$ & 0 \\
Resección de tumor pleural & 1 & $1(100 \%)$ & 0 & 0 \\
\hline
\end{tabular}

Los porcentajes hacen referencia al mismo procedimiento quirúrgico. N: número de pacientes sometidos a la determinada intervención quirúrgica; PAl: monitorización de presión arterial invasiva mediante catéter radial; SV: sondaje vesical; CVC: catéter venoso central.

pacientes (42,5\%), S en $29(39,7 \%)$, S+l en $12(16,4 \%)$ y $E+l$ en 1 paciente $(1,4 \%)$. El consumo de fentanilo ajustado por peso (mcg/kg) fue 6,7 (DE 2,9) en el grupo l, 4,6 (DE 1,4) en el grupo $S, 4,5(D E 1,4)$ en el grupo $S+l$ y 6,5 en el grupo $E+l$. En global, el consumo medio fue de $5,5 \mathrm{mcg} / \mathrm{kg}$. S y $\mathrm{S}+\mathrm{l}$ requirieron menos consumo de fentanilo comparado con el resto de los otros grupos ( $p$-valor $=0,01$ ).

Los fármacos analgésicos empleados intraoperatoriamente y sus coadyuvantes se encuentran en la Tabla 3.

\section{Destino postoperatorio y estancia hospitalaria}

Todos los pacientes fueron extubados en el quirófano. Cuarenta y ocho pacientes $(65,7 \%)$ fueron trasladados a la UCC, con una media de 21,1 (DE 5,2) horas de ingreso. Veinticinco pacientes $(34,2 \%)$ se trasladaron a URPA para, posteriormente, 22 de ellos $(30,1 \%)$ ingresar en planta de hospitalización de cirugía torácica y $3(4,1 \%)$ ser dados de alta el mismo día en régimen de CMA. Entre las timectomías 9 ingresaron en unidad de críticos posquirúrgicos, con una media de 17,6 (DE 2,7) horas de ingreso, 7 ingresaron en URPA y posteriormente planta de hospitalización y 3 pacientes entraron en el circuito de CMA. 32 de los 33 casos de lobectomía ingresaron en la UCC con 21,6 $(D E 3,9)$ horas de ingreso, y 1 fue de URPA a planta. Cuatro de las 17 resecciones en cuña ingresaron en UCC, con 21,4 (DE 4,8 ) horas de ingreso, y 13 fueron de URPA a planta. El caso de lobectomía y resección en cuña, así como los dos casos de timectomía y resección en cuña, ingresaron en unidad de críticos posquirúrgicos, con una media de 18,8 h y 28,8 (DE 21,0 ) horas de ingreso, respectivamente.

Se produjo un único reingreso en unidad de críticos, por isquemia intestinal en planta, el día 6 del postoperatorio. La media de días de ingreso fue 4,7 (DE 5,7). Analizada la estancia hospitalaria según el tipo de intervención quirúrgica, ésta fue de 1,7 días en las timectomías (DE 1,3); 7,4 en las lobectomías (DE 7,3), 2,6 (DE 1,0) días para los casos de resección en cuña; 2 días en el caso de lobectomía más resección en cuña, 1 día en el caso de resección de tumor pleural y 9 días (DE 7) en los dos pacientes en quienes se realizó timectomía junto con resección en cuña.

\section{Dolor postoperatorio}

La media de dolor postoperatorio, evaluado mediante NPRS, a la 1, 24 y $48 \mathrm{~h}$ postoperatorias fue de 2,8 (DE 2,6), 1,7 $(D E 1,8)$ y $1(D E 1,4)$, respectivamente. Por grupos de técnica analgésica; los pacientes del grupo I puntuaron de media 2,6 (DE 2,4), 1,5 (DE 1,7) y $0,8(1,4)$ a la $1 \mathrm{~h}$. Los pacientes del

\begin{tabular}{llc}
\multicolumn{2}{l}{ Tabla 3. Abordaje multimodal farmacológico intraoperatorio } \\
\hline \multirow{3}{*}{ Opioides } & Fentanilo & $\mathbf{n}(\%)$ \\
& Remifentanilo & $73(100 \%)$ \\
Analgésicos & Paracetamol & $3(4,1 \%)$ \\
convencionales & Dexketoprofeno & $73(100 \%)$ \\
& Metamizol & $48(65,8 \%)$ \\
Adyuvantes & Corticoesteroides & $34(46,6 \%)$ \\
& Sulfato de magnesio & $18(24,7 \%)$ \\
& Ketamina & $20(27,4 \%)$ \\
& & $3(4,1 \%)$ \\
\hline
\end{tabular}

grupo S puntuaron un 2,8 (DE 2,4), 1,6 (DE 1,6) 1,1 (DE 1,2). Los del grupo $\mathrm{S}+1$ puntuaron con 4,3 (DE 3,9), 2,5 (DE 2,4) y 1,0 (DE 1,6). El único paciente $E+1$ puntuó 3 tras una hora de la cirugía, 3 tras 24 h y 5 pasadas $48 \mathrm{~h}$. No se encontraron diferencias estadísticamente significativas entre la técnica locorregional empleada y la puntuación NPRS para ninguno de los momentos registrados $(p$-valor $=0,71, p$-valor $=0,32, p$-valor $=$ 0,19 respectivamente).

En la UCC, el dolor fue tratado mediante bolos de morfina intravenosa administrados por enfermería en 39 pacientes $(54,1 \%)$ y bolos de morfina administrados mediante analgesia controlada por el paciente (PCA) en 14 casos $(19,4 \%)$, además de paracetamol y antiinflamatorios no esteroideos.

\section{Complicaciones postoperatorias}

Aparecieron náuseas y vómitos posoperatorios en 22 casos $(30,5 \%)$ en las primeras $24 \mathrm{~h}$ del posoperatorio. Se registraron un total de 7 casos $(9,5 \%)$ de fugas persistentes a través del drenaje torácico, 2 casos $(2,7 \%)$ de enfisema subcutáneo, 1 caso $(1,4 \%)$ de necesidad de reintubación en el postoperatorio inmediato en la UCC y $1(1,4 \%)$ caso de éxitus a los 11 días de la cirugía por isquemia intestinal masiva, diagnosticada e intervenida el día 6 del posoperatorio.

\section{Discusión}

Hasta donde sabemos, se trata del primer estudio que describe resultados perioperatorios de cirugía torácica asistida por robot en España. En lo referente a la técnica anestésica, muy poco es lo descrito previamente[9-[12].

En líneas generales, nuestros resultados muestran que los 
pacientes sometidos a cirugía torácica robótica son extubados en quirófano, presentan una corta estancia en UCC y hospitalaria, así como una escasa tasa de complicaciones postoperatorias.

Un buen colapso del pulmón no dependiente es fundamental en los procedimientos RATS para minimizar la presión de insuflación de $\mathrm{CO}_{2}$ y mejorar la visualización del parénquima pulmonar o estructuras mediastínicas por parte del equipo quirúrgico[9]. En nuestra experiencia, comparando la RATS con la VATS y los abordajes mediante toracotomía, la deflación pulmonar es más dificultosa por el pequeño tamaño de las incisiones portales del robot y la ausencia de comunicación de la cavidad torácica con el medio ambiente como sucede con la VATS y la toracotomía. Por lo tanto, el dispositivo de aislamiento pulmonar se debe escoger en base a la capacidad para asegurar una óptima deflación pulmonar y mayor capacidad de aspiración del pulmón no dependiente. Tradicionalmente en nuestro centro, siempre que sea posible en RATS se lleva a cabo la ventilación unipulmonar mediante tubos de doble luz.

Los resultados de la monitorización invasiva empleada en RATS en nuestro centro dependen en gran medida del tipo de intervención quirúrgica. Tan solo se cateterizó un acceso venoso central, cosa que va a favor de la menor invasividad anestésica en este tipo de abordaje quirúrgico mínimamente invasivo.

La RATS presenta resultados prometedores en cuanto a la reducción de dolor agudo postoperatorio por tratarse de una técnica mínimamente invasiva[4],[13]-[15]. El abordaje multimodal del dolor en la cirugía torácica debe considerarse mandatorio debido a las múltiples aferencias sensitivas tras la agresión quirúrgica[5],[16], con el objetivo de reducir al máximo las potenciales complicaciones del dolor agudo postoperatorio, así como la cronificación del dolor[11],[15],[16]. La técnica regional por excelencia en la toracotomía ha sido, tradicionalmente, la analgesia epidural torácica[17]. Recientemente, se han descrito los beneficios de los bloqueos de la pared torácica para cirugía del tórax como cirugía mamaria, cardíaca y torácica, sin embargo, con falta de datos en su idoneidad para la cirugía robótica torácica[18]-[23].

En nuestro centro, la analgesia intraoperatoria comprende opioides, analgésicos, antiinflamatorios esteroideos y no esteroideos y otros adyuvantes, como la ketamina o el sulfato de magnesio, junto a técnicas locorregionales. Se entendieron las ventajas del bloqueo del plano serrato respecto otros bloqueos de la pared torácica por la facilidad de localización del espacio de punción tras la colocación del paciente en decúbito lateral, por haber referencia ósea clara ( $5^{\mathrm{a}}$ costilla) así como el fácil direccionamiento de la aguja[20]. No se colocaron catéteres epidurales ni paravertebrales para reducir la invasividad, y favorecer una movilización precoz del paciente[24]. Se observó que los pacientes en quienes se realizó este bloqueo $(S)$ requirieron una menor dosis de fentanilo intraoperatorio que aquellos en quienes no se realizó, lo cual debería estudiarse de manera prospectiva y aleatorizada ya que este estudio no se diseñó para tal objetivo.

Por la mayor frecuencia la aparición de problemas respiratorios, ingresaron en UCC prácticamente la totalidad de pacientes con resecciones lobares[25], mientras que los pacientes intervenidos de resección en cuña o de timectomía tuvieron índices de ingreso en esta unidad mucho menores $(23,5 \%$ y $47,3 \%$, respectivamente). Durante el primer año de implementación del programa de cirugía torácica robótica tan sólo 3 pacien- tes $(4,1 \%)$ operados de timectomía fueron tratados con circuitos clínicos de CMA. No obstante, y después de la experiencia obtenida creemos que se deben intensificar los esfuerzos para incrementar el número de pacientes tratados de forma ambulatoria para beneficiar al paciente acelerando su recuperación a la vez que se disminuye el consumo de recursos. La estancia hospitalaria media de 5 días obtenida en esta serie se corresponde con el de otros grupos[4] bajo el paraguas de un programa multidisciplinar de recuperación acelerada después de cirugía robótica. Estos programas de recuperación acelerada incluyen generalmente conseguir un adecuado control del dolor postoperatorio para promover una pronta movilización, iniciar de forma precoz la tolerancia por vía oral y disminuir las posibles complicaciones pulmonares al permitir la rehabilitación respiratoria[6].

En cuanto al dolor postoperatorio, los resultados muestran un dolor menor de NPRS 3 transcurridas tanto 1 hora del postoperatorio como a las 24 y $48 \mathrm{~h}$, por lo que consideramos un adecuado control del dolor tras la intervención con las medidas empleadas. En nuestro trabajo, las diferentes técnicas regionales no tuvieron un impacto en la disminución del dolor postoperatorio en ninguno de los momentos observados, aunque la literatura sugiere que el bloqueo del plano serrato anterior reduce el dolor por lo menos en las primeras 12 h[19],[20].

La tasa de complicaciones posoperatorias registradas en este estudio se corresponde a otras series[4]. La aparición de NVPO no se relacionó con la técnica analgésica ni con la cantidad de opioides intraoperatorios administrados.

Las limitaciones fundamentales de este estudio son su diseño, de carácter retrospectivo y los sesgos derivados de ello, la ausencia de un grupo control y la participación de un único centro en la generación y recogida de datos.

A partir de nuestras observaciones, creemos que deben desarrollarse estudios prospectivos, aleatorizados, para analizar la reducción de mórficos intraoperatorios con la estancia hospitalaria o reducción de complicaciones postoperatorias y la relación entre la analgesia multimodal y la cronificación del dolor agudo postoperatorio.

Como conclusión, en nuestra experiencia, la RATS es una técnica segura, habiendo registrado escasas complicaciones intraoperatorias y postoperatorias, que permite una corta estancia en unidad de críticos cuando ésta se considera a la vez que una pronta alta a domicilio; en algunos casos seleccionados de timectomía, incluso, permitiendo el régimen de cirugía mayor ambulatoria. Así como las técnicas quirúrgicas tienden a una menor invasividad, la técnica anestésica debe ir de la mano, por lo que los bloqueos de la pared torácica abren en este sentido un gran abanico para la investigación. En nuestro estudio observamos que el bloqueo del plano serrato anterior disminuyó la necesidad de fentanilo intraoperatorio, cuyo significado clínico debería estudiarse con más profundidad en futuros estudios multicéntricos, prospectivos y aleatorizados.

Agradecimientos: Damos las gracias a Marta Larroya, MD, por su magnífica labor con la estadística del estudio.

\section{Referencias}

1. Mungo B, Hooker CM, Ho JSY, Yang SC, Battafarano RJ, Brock 
M V., et al. Robotic Versus Thoracoscopic Resection for Lung Cancer: Early Results of a New Robotic Program. J Laparoendosc Adv Surg Tech. 2016;26(4):243-8. https://doi.org/10.1089/ lap.2016.0049 afa CC, Romano G, Key TH, Davini F, Melfi F. The evolution of robotic thoracic surgery. Ann Cardiothorac Surg. 2019;8(2):210-7. https://doi.org/10.21037/acs.2019.03.03

3. Duclos G, Charvet A, Resseguier N, Trousse D, D'Journo XB, Zieleskiewicz $L$, et al. Postoperative morphine consumption and anaesthetic management of patients undergoing video-assisted or robotic-assisted lung resection: A prospective, propensity score-matched study. J Thorac Dis. 2018;10(6):3558-67. https:// doi.org/10.21037/jtd.2018.05.179

4. Kwon ST, Zhao L, Reddy RM, et al. Evaluation of acute and chronic pain outcomes after robotic, video-assisted thoracoscopic surgery, or open anatomic pulmonary resection. J Thorac Cardiovasc Surg. 2017;154(2):652-659.e1 https://doi.org/10.1016/j. jtcvs.2017.02.008

5. Hazelrigg SR, Cetindag IB, Fullerton J. Acute and chronic pain syndromes after thoracic surgery. Surg Clin North Am. 2002;82(4):849-65. https://doi.org/10.1016/S00396109(02)00031-2

6. Piccioni F, Ragazzi R. Anesthesia and analgesia: how does the role of anesthetists changes in the ERAS program for VATS Iobectomy. J Vis Surg. 2018;4(5):9-9. https://doi.org/10.21037/ jovs.2017.12.11

7. Umari M, Falini S, Segat M, Zuliani M, Crisman M, Comuzzi L, et al. Anesthesia and fast-track in video-assisted thoracic surgery (VATS): From evidence to practice. J Thorac Dis. 2018;10(1): S542-54. https://doi.org/10.21037/jtd.2017.12.83

8. Von Elm E, Altman DG, Egger M, Pocock SJ, Gøtzsche PC, Vandenbroucke JP. The Strengthening the Reporting of Observational Studies in Epidemiology (STROBE) Statement: Guidelines for reporting observational studies. UroToday Int J. 2009;2(2):10-4.

9. McCall P, Steven M, Shelley B. Anaesthesia for video-assisted and robotic thoracic surgery. BJA Educ. 2019;19(12):405-11. https:// doi.org/10.1016/j.bjae.2019.09.002

10. Pauli H, Eladawy M, Park J. Anesthesia for robotic thoracic surgery. Ann Cardiothorac Surg. 2019;8(2):263-8. https://doi. org/10.21037/acs.2018.12.06

11. Heller JA, Bhora FY, Heller BJ, Cohen E. Robotic-assisted thoracoscopic lung surgery: Anesthetic impact and perioperative experience. Minerva Anestesiol. 2018;84(1):108-14. https://doi. org/10.23736/S0375-9393.17.12168-1

12. Bussières JS, Somma J, del Castillo JLC, Lemieux J, Conti M, Ugalde PA, et al. Comparaison du bloqueur bronchique à la sonde endotrachéale à double lumière gauche en chirurgie thoracoscopique vidéoassistée: une étude randomisée contrôlée examinant le temps et la qualité de l'affaissement du poumon. Can J Anesth. $2016 ; 63(7): 818-27$. https://doi.org/10.1007/s12630-016-0657-3

13. Ghee, C, Vigneswaran W. Robot assisted thoracic surgery: a review of current literature. Ann Cardiovasc Thorac Surg. 2018;01(03):71-5. https://doi.org/10.35841/cardiovascular-surgery.1.3.71-69

14. Crumley S, Schraag S. The role of local anaesthetic techniques in ERAS protocols for thoracic surgery. J Thorac Dis. 2018;10(3):1998-2004. https://doi.org/10.21037/jtd.2018.02.48

15. Kehlet $\mathrm{H}$, Jensen TS, Woolf $\mathrm{CJ}$. Persistent postsurgical pain: risk factors and prevention. Lancet. 2006;367(9522):1618-25. https:// doi.org/10.1016/S0140-6736(06)68700-X

16. Fiorelli S, Cioffi L, Menna C, et al. Chronic Pain After Lung Resection: Risk Factors, Neuropathic Pain, and Quality of Life. J Pain Symptom Manage. 2020;60(2):326-335. https://doi. org/10.1016/j.jpainsymman.2020.03.012

17. Rawal N. Epidural Technique for Postoperative Pain. Reg Anesth Pain Med. 2012;37(3):310-7. https://doi.org/10.1097/ AAP.0b013e31825735c6

18. Batchelor TJP, Rasburn NJ, Abdelnour-Berchtold E, et al. Guidelines for enhanced recovery after lung surgery: recommendations of the Enhanced Recovery After Surgery (ERAS $®$ ) Society and the European Society of Thoracic Surgeons (ESTS). Eur J Cardiothorac Surg. 2019;55(1):91-115. https://doi.org/10.1093/ejcts/ezy301

19. De Cassai A, Boscolo A, Zarantonello F, et al. Serratus anterior plane block for video-assisted thoracoscopic surgery: A meta-analysis of randomised controlled trials. Eur J Anaesthesiol. 2021;38(2):106-114. https://doi.org/10.1097/ EJA.0000000000001290

20. Viti A, Bertoglio P, Zamperini M, Tubaro A, Menestrina N, Bonadiman $S$, et al. Serratus plane block for video-assisted thoracoscopic surgery major lung resection: A randomized controlled trial. Interact Cardiovasc Thorac Surg. 2020;30(3):366-72. https://doi. org/10.1093/icvts/ivz289

21. Khalil AE, Abdallah NM, Bashandy GM, Kaddah TA. UltrasoundGuided Serratus Anterior Plane Block Versus Thoracic Epidural Analgesia for Thoracotomy Pain. J Cardiothorac Vasc Anesth. 2017;31(1):152-158. https://doi.org/10.1053/j.jvca.2016.08.023

22. Urits I, Ostling PS, Novitch MB, Burns JC, Charipova K, Gress KL, et al. Truncal regional nerve blocks in clinical anesthesia practice. Best Pract Res Clin Anaesthesiol. 2019;33(4):559-71. https://doi. org/10.1016/j.bpa.2019.07.013

23. Chin KJ, Pawa A, Forero M, Adhikary S. Ultrasound-Guided Fascial Plane Blocks of the Thorax: Pectoral I and II, Serratus Anterior Plane, and Erector Spinae Plane Blocks. Adv Anesth. 2019; 37:187-205. https://doi.org/10.1016/j.aan.2019.08.007

24. Rawal N. Current issues in postoperative pain management. Eur J Anaesthesiol. 2016;33(3):160-71. https://doi.org/10.1097/ EJA.0000000000000366

25. Lugg ST, Agostini PJ, Tikka T, Kerr A, Adams K, Bishay E, et al. Long-term impact of developing a postoperative pulmonary complication after lung surgery. Thorax. 2016;71(2):171-6. https:// doi.org/10.1136/thoraxjnl-2015-207697 